\title{
L'eroe cinese von Pietro Metastasio und Johann Adolf Hasse, Warschau 1754
}

\section{Pietro Metastasio's and Johann Adolf Hasse's L'eroe cinese in Warsaw (1754)}

\author{
Alina Żórawska-Witkowska / azorwit@wp.pl \\ Institute of Musicology, University of Warsaw, PL
}

\begin{abstract}
L'eroe cinese is a very important work in the history of operatic theatre in Warsaw (that was a cultural centre of the Polish-Lithuanian Commonwealth) as it is the first opera seria in the strict sense of the term that was staged here and the first of a series of Johann Adolf Hasse's opere serie staged subsequently between 1759 and 1763 in the King's Opernhaus of August III in Warsaw (the theatre itself was opened in 1748). It is probable that 1754 performance was led by the composer himself. The opera was accompanied by ballets choreographed by Antoine Pitrot, including the concluding ballet chinois pantomime (possibly a ballet d'action, thus one of the earliest examples of the genre) and L'adoration de la pagode. L'eroe cinese was, therefore, of particular importance for the theatrical and literary culture of Polish-Lithuanian Commonwealth. The libretto by Metastasio was printed in Warsaw in its original Italian version as well as Italian-French and Italian-German editions. Moreover, in 1755 a Polish translation Bohatyr chiński was issued in print. To stage the opera in Warsaw in honour of August III's birthday (7 October), the 150-strong artistic and technical personnel serving Dresden Electoral Court moved to the Polish capital. Amongst the numerous spectators of the eight performances were not only the King and the Queen, their two sons and almighty Minister Heinrich von Brühl but also aristocratic elite of the Commonwealth including the future King Stanisław August Poniatowski. The focus of the paper is placed on the special circumstances of Warsaw staging of L'eroe cinese, then the Author deals with ideological overtones and form of the Metastasian libretto (Vienna, 1752) as well as its musical setting by Hasse (Hubertusburg, 1753), in both cases Warsaw version bearing minor differences from original settings.
\end{abstract}




\section{Keywords}

1754, king August III, Warsaw, opera seria, L'eroe cinese, Pietro Metastasio, Johann Adolf Hasse, Antoine Pitrot, Teresa Albuzzi Todeschini, Pasquale Bruscolini, Bartolomeo Putini, Giuseppe Belli, Ludwig Cornelius

L'eroe cinese von Pietro Metastasio und Johann Adolf Hasse ist die erste Opera seria sensu stricto, die in Warschau, der Hauptstadt Polens, aufgeführt wurde. Dramma per musica war hier zwar schon in der ersten Hälfte des 17. Jahrhunderts (1635-1648) am Hof des Königs Ladislaus' IV. Wasa intensiv gepflegt worden, ${ }^{1}$ aber dann wurden nur noch vereinzelte und seltene Beispiele in Polen präsentiert. Selbstredend war die Opera seria als Gattung den Dresden besuchenden oder auch Europa bereisenden Magnaten gut bekannt, bisweilen reproduzierten sie diese Operngattung sogar an ihren eigenen Höfen.

Zur Aufführung der L'eroe cinese, einem denkwürdigen Ereignis für die Kultur der Republik Beider Nationen (der Rzeczpospolita Obojga Narodów), kam es 1754, also unter der Herrschaft Augusts III., des Königs von Polen (1734-1763) und gleichzeitig Kurfürsten von Sachsen (1733-1763). Die Doppelherrschaft verlangte von August III., dass er seine Anwesenheit, seine Pflichten und sein künstlerisches Personal zwischen beiden Staaten teilte, aber diese Teilung asymmetrisch war, selbstredend zugunsten Sachsens. ${ }^{2}$ Nichtsdestoweniger profitierte die Stadt Warschau, wo der königliche Hof während der Aufenthalte in Polen residierte, in der Sphäre des Theater- und Musiklebens enorm. Das kam letztlich auch den Magnaten der Adelsrepublik zugute, die an ihren Höfen eigene künstlerische Zentren gründeten.

Einen entscheidenden Meilenstein in Warschaus Theaterlandschaft markierte das Jahr 1748, als am 3. August während des bereits sechsten Aufenthalts Augusts III. in der polnischen Hauptstadt das neue königliche Theater eröffnet wurde. Das auf Kosten des sächsischen Hofs errichtete Theater war so angelegt, dass es für die Darbietung von Opere serie geeignet war. Zutritt hatten Edelgeborene, königlich-kurfürstliche Beamte und Künstler, Priester und ausgewählte Bürger, die vom Oberhofmarschallamt kostenlose Eintrittskarten erhielten. ${ }^{3}$

Nun verfügte August III. in Warschau also über ein entsprechend ausgestattetes Operntheater. Daher fasste er den Beschluss, den hiesigen Untertanen die ihnen nicht

1 Siehe PRZYBYSZEWSKA-JARMIŃSKA, Barbara. The Baroque, part 1: 1595-1696, (vol. 3 of The History of Music in Poland). Warsaw: Sutkowski Edition, 2002, S. 395ff; ŻÓRAWSKA-WITKOWSKA, Alina. Dramma per musica at the Court of Ladislaus IV Vasa (1627-1648). In Italian Opera in Central Europe, vol. I: Institutions and Ceremonies. Melania Bucciarelli - Norbert Dubowy - Reinhard Strohm (eds.). Berlin: Berliner WissenschaftsVerlag, 2006.

2 ŻÓRAWSKA-WITKOWSKA, Alina. Muzyka na polskim dworze Augusta III [Musik am polnischen Hof Augusts III.], I. Teil, Lublin: Wydawnictwo Muzyczne Polihymnia, 2012, S. 57 ff.

3 Siehe w.o., S. 113 ff. In D-Dla, OHMA G Nr. 57 b Italienische Komödien, so auf dem Königl. Hoftheater in Warschau aufgeführet worden 1748, 1749, OHMA G Nr. 61 b Opern und Italiänischen Comedien so auf dem Königl: Hof-Theatro in Warschau aufgeführet worden in Ao: 1754, OHMA Nr. 66 c Opern so auf dem Königl. Hof-Theatro in Warschau aufgeführet worden in Annis 1759. 1760. 1761. et 1762, sind sowohl Listen der Personen, die Eintrittskarten erhielten, als auch Musteroriginale der Eintrittskarten selbst überliefert. 
weiter bekannte, aber von ihm hoch geschätzte Gattung Dramma per musica/Opera seria nahe zu bringen. Diesen Beschluss verwirklichte er erst im Herbst 1754 während seines schon neunten Aufenthalts in Polen. Westeuropa schickte sich zu diesem Zeitpunkt bereits an, diese stagnierende Gattung, als deren Großmeister Pietro Metastasio, der poeta cesareo, und Johann Adolf Hasse, der Kapellmeister des polnisch-sächsischen Hofs galten, zu reformieren. Die Aufführung des Dramma per Musica L'eroe cinese von Metastasio und Hasse 1754 im königlichen Theater in Warschau ist in den Quellenüberlieferungen ziemlich gut dokumentiert.

\section{Umstände der Aufführung}

Die fast sechsmonatige Anwesenheit des Königs in der zweiten Hälfte des Jahres 1754 (21. Juni - 16. Dezember) in Warschau brachte es mit sich, dass eine größere Gruppe von Künstlern und Theaterhandwerkern als je zuvor von Dresden nach Warschau aufbrach. Dieses Mal nämlich sollten nicht nur italienische Komödien, sondern auch das erste - wie ich schon hervorgehoben habe - Opera seria in der bereits zwanzigjährigen Königsherrschaftszeit Augusts III. aufgeführt werden. Alle Vorstellungen sollten von Balletten begleitet werden. Nach Warschau kamen von Dresden also weit über 150 Künstler sowie autres personnes employées au Theatre, darunter der für die gesamten Theaterangelegenheiten des Hofes verantwortliche Directeur des Plaisirs Carl Heinrich von Dieskau; eine Opernsängerin und vier Opernsänger - Teresa Albuzzi Todeschini, Pasquale Bruscolini, Bartolomeo Putini, Giuseppe Belli, Ludwig Cornelius; ca. 30 Musiker aus den königlichen Kapellen, das heißt der Dresdner und der polnischen, sowie auch Mitglieder der Kapelle des Ministers Heinrich von Brühl; 14 comici italiani (das ganze Ensemble); 28 Tänzer (das gesamte Ballettensemble); zwei Komponisten - Johann Michael Breunich (Kirchen-Componist) und Johann Adam (Komponist der Ballettmusik), die von ihren Kopisten begleitet wurden; der zu den Ballettproben aufspielende Geiger François de Francini; 7 Hoftrompeter und 1 Pauker (ein Teil des Ensembles); 4 Bockpfeiffer (ein Teil der Truppe); der deutsche Dichter und Hofnarr Haentzschel. „Verschiedene andere Persohnen“ waren u. a.: der Theaterinspekteur Antonio Maria Cattaneo, 6 Theater Bedienten, 1 valet de la Danse, 3 Theater-Mahler, der Perückenmacher und sein Geselle, 2 Schneider-Meister und 10 Gesellen, 1 Land-Bau-Meister, 2 Beigefühlen bei der Garderobe; 1 Bau-Schreiner, 1 Sprizen-Meister, 1 Wagen-Meister, 30 Zimmerleute, 16 FeuerWächter, 12 Kutscher, 6 Persohnen von der Beleuchtung. ${ }^{4}$

In den mir bekannten Quellen habe ich hingegen keine Spur der Anwesenheit weder des Kapellmeisters Johann Adolf Hasse noch des Theaterarchitekten Giuseppe Galli Bibiena in Warschau gefunden. Letzterer sollte angeblich das Bühnenbild für die

4 ŻÓRAWSKA-WITKOWSKA, Alina. Between Dresden and Warsaw: The Travels of the Court of August III of Poland (Friedrich August II of Saxony). In Musicology Today: Polish Studies on Baroque Music, Szymon Paczkowski - Anna Ryszka-Komarnicka (eds.). Warsaw: Institut of Musicology University of Warsaw, 2009, p. 19-20. 
Warschauer L'eroe cinese entworfen haben ${ }^{5}$. Von den fünf von mir angenommenen Aufenthalten Hasses in Warschau ${ }^{6}$ - und wie Charles Burney feststellte, „begleitete [Hasse] diesen Herrn [das ist August III.] sehr oft nach Warschau wo er verschiedene Opern komponierte ${ }^{\text {"7 }}$ - ist nur ein Aufenthalt durch Quellen belegt (1762-1763). Ich gehe jedoch davon aus, dass Hasses Anwesenheit im Herbst 1754 in Warschau nicht nur möglich (es gibt keinen Hinweis auf eine Aktivität des Komponisten in der fraglichen Zeit an einem anderen Ort), sondern geradezu notwendig gewesen war, damit er die Premiere seiner ersten Oper in Polen, noch dazu in der Interpretation von größtenteils anderen Solisten als bei der Uraufführung (Hubertusburg, 7. Oktober 1753) vorbereiten konnte.

Die Theatersaison 1754 wurde am 11. September eröffnet und am 27. November mit Beginn des Advents abgeschlossen. Ausgefüllt wurde sie mit zwei oder drei Mal pro Woche aufgeführten italienischen Komödien. Seit dem 7. Oktober wurden die Premiere und sieben Wiederholungen der Oper zwischengeschaltet. Alle Vorstellungen wurden von je 3 Balletten begleitet, passend zu jedem der drei Akte der dargebotenen Werke. Insgesamt kamen während der 20 Theaterabende damals 9 Komödien dell'arte und 3 literarische Komödien zur Aufführung (ihre Autoren waren Girolamo Focher, Cesare d'Arbes und Carlo Goldoni), achtmal wurde das Dramma per musica L'eroe cinese dargeboten, darüber hinaus wurden einige Dutzend (ca. 60) Ballette präsentiert. ${ }^{8}$

Die Uraufführung der L'eroe cinese von Hasse am 7. Oktober 1753 in Hubertusburg verlieh dem Geburtstag Augusts III., der gewöhnlich im dortigen Jagdschloss gefeiert wurde, einen festlichen Rahmen. Auch die Warschauer Premiere ein Jahr später fand aus demselben Anlass statt, also am 7. Oktober 1754. Minister Heinrich von Brühl schrieb an diesem Tag dem in Sachsen weilenden Minister Joseph Anton Gabaleon von Wackerbarth-Salmour: „Ihre Königliche Hoheit [Maria Josepha] schenkt ihrem königlichen Gatten ein schönes Bouquet, nämlich die Oper L'eroe cinese, die an diesem Abend gespielt wird

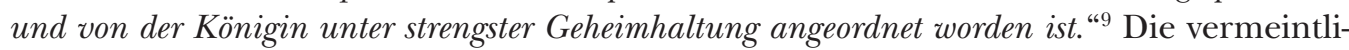
che Geheimhaltung musste es eine gängige Redewendung sein.

Bei der Uraufführung von Hasses L'eroe cinese in Hubertusburg traten exzellente Sänger aus der in Dresden residierenden königlich-kurfürstlichen Kapelle auf, nämlich der Tenor Angelo Maria Amorevoli (Leango) sowie die Soprane Angelo Maria Monticelli (Siveno), Giuseppe Belli (Minteo), Teresa Albuzzi-Todeschini (Lisinga) und Caterina Pilaja (Ulania). ${ }^{10}$ In Warschau wurde die Besetzung verändert. Nur Albuzzi-Todeschini

5 Eine solche Vermutung äußerte MELLACE, Raffaele. Johann Adolf Hasse. Palermo: L'Epos, 2004, S. 90; Idem. Johann Adolf Hasse, neubearbeitete Ausgabe, Übersetzung Juliane Riepe, Beeskow: ortus musikverlag, 2016, S. 72.

6 ŻÓRAWSKA-WITKOWSKA, Alina. Johann Adolf Hassse und die Musik am polnischen Hof Augusts III. (1734-1763). In Hasse-Studien 7, Wolfgang Hochstein - Reinhard Wiesend (hrsg.). Stuttgart: Carus Verlag, 2012, S. 61-68.

7 BURNEY, Carl. Tagebuch einer Musikalischen Reise, Bd. 2: Durch Flandern, die Niederlande und am Rhein bis Wien. Hamburg: Bode, 1773, S. 257.

8 Siehe ŻÓRAWSKA-WITKOWSKA, Alina. Muzyka na polskim dworze, S. 445 ff.

9 D-Dla, Geheimnis Kabinett, Loc. 720, Correspondance tenue durant le séjour de la cour en Pologne du Comte de Wackerbarth avec le comte de Brühl. 1754, Brief vom 7. Oktober 1754, im Original in französischer Sprache.

10 METASTASIO, Pietro. L'eroe cinese, Vedova Stössel, Dresda 1753, nicht paginierte Seite. 
sowie Belli wiederholten ihre Rollen, die übrigen Interpreten präsentierten sich in dieser Oper zum ersten Mal - der Tenor Ludwig Cornelius (Leango), die Kastraten Pasquale Bruscolini (Siveno) und Bartolomeo Putini (Ulania). ${ }^{11}$ An der Aufführung waren auch zahlreiche Statisten (comparse) wie paggi cinesi, paggi tartari, schiave tartare, manderini d'armi, manderini di lettere, bonzi, soldati cinesi beteiligt. Auch die in den Warschauer Opernaufführungen auftretenden Sänger waren brillante Künstler. Sie alle hatten ihre europäische Karriere im fünften Jahrzehnt des 18. Jahrhunderts begonnen. ${ }^{12}$

Die Warschauer Premiere der L'eroe cinese bildete nur einen Teil, wenn auch zweifellos den wichtigsten, der gesamten Geburtstagsgala des Königs. Im höfischen Ritual war festgelegt, wie die Festveranstaltung abzulaufen hatte. So kündigten am frühen Morgen donnernde Kanonensalven allen Einwohnern der Stadt jenen Freudentag an; am Vormittag nahm der König in seiner Residenz, dem Sächsischen Palais, Glückwünsche entgegen, danach nahm er zusammen mit seiner Familie an einer in der dortigen Kapelle abgehaltenen Messe teil; während des im Sächsischen Palais veranstalteten Festmahls wurden Trinksprüche ausgebracht, begleitet von Kanonensalven sowie Fanfaren von Trompeten und Pauken; um 16 Uhr begann in der Operalnia/Opernhaus die Aufführung der L'eroe cinese. Wie die Warschauer Presse bekannt gab, war der Sächsische Garten, wo sich das Theater befand, „mit wunderschönen Säulen, mit verschiedenen auf Sockeln gemalten Festons dekoriert und erstrahlte im üppigen Licht der Öllampen"13. Im Theater wurde der König mit Fanfaren von Trompeten und Pauken begrüßt. Nach der Aufführung begab er sich zusammen mit den Zuschauern auf einen Spaziergang durch den prächtig illuminierten Garten.

Für die Uraufführung waren 1.079 Eintrittskarten verteilt worden. ${ }^{14}$ Das Theater bot aber eigentlich nur ca. 560 Zuschauern Platz. Daher herrschte ein riesiges Gedränge, und die Königin Maria Josepha teilte ihrer Schwiegertochter Maria Antonia Walpurgis, der Frau des Prinzen Friedrich Christian, mit: „Pünktlich um 16:00 sind wir in die Oper gegangen, wo wegen der großen dort anwesenden Menschenmenge eine entsetzliche Hitze herrschte. Eine solche Aufführung hatte man in Warschau noch nie zuvor gesehen. Die Interpretation war höchst gelungen und Putini war eine sehr schöne junge Dame [als Ulania]. Während der Oper hatte ich befohlen, unseren großen [Garten] und zwei kleine Gärten vor unseren Fenstern zu illuminieren. " ${ }^{15}$ Auch den Jubilar hatte die Interpretation der Oper höchst zufrieden gestellt, wovon er seinem Sohn Friedrich Christian in dem ihm eigenen lakonischen Stil berichtete: „Die Oper ist sehr gut gelungen und alles wurde sehr gut gesungen und la seconda

11 METASTASIO, Pietro. L'eroe cinese. Varsavia: Stamperia Regia e della Repubblica delle Scuole Pie, 1754, nicht paginierte Seite. Betont sei, dass dies der einzige Fall ist, da im unter August III. in Warschau gedruckten Libretto die Namen der Sänger genannt wurden.

12 Ihre Karrieren wurden genauer in ŻÓRAWSKA-WITKOWSKA Alina, Muzyka na polskim dworze, S. 337 ff präsentiert.

13 „Kurier Polski” 1754 Nr. 64, nicht paginierte Seite; im Original in polnischer Sprache.

14 D-Dla, OHMA G Nr 61 b.

15 D-Dla, Nachlass Maria Antonia Nr 17, Brief Maria Josephas an Maria Antonia, Warschau, 10. Oktober 1754; Original des Briefs in französischer Sprache. 
Donna S.a Barthlomea [Bartolomeo Putini] ist eine sehr gute Komparsin “. ${ }^{16}$ Selbst der Minister von Brühl berichtete Maria Antonia Walpurgis, dass die Dekorationen sehr gut geworden seien, dass die dem Orchester nicht förderliche Akustik des Theaters sich als äußerst günstig für die Sänger erwiesen habe und die geräumige Bühne es den Tänzern erlaubte, ihre ganze Kunst unter Beweis zu stellen. ${ }^{17}$

Unter dem Premierenpublikum der Oper befanden sich zahlreiche polnische und litauische Magnaten, darunter Stanisław Antoni Poniatowski, damals Starost von Przemyśl und später als Stanislaus Augst König von Polen. Poniatowski sah die Oper L'eroe cinese mindestens dreimal. Für die einzelnen Vorstelllungen wurden von über 1000 bis über 1200 Eintrittskarten verteilt. Wiewohl war es einmal geschehen, dass noch wesentlich mehr Leute erfolglos versucht hatten, ins Theater hineinzugelangen.

Die warschauer Premiere der Oper wurde von Balletten begleitet. Es waren: zum I. Akt Ballet des bergers serieux; zum II. Akt Ballet serieux; nach dem III. Akt wurde zuerst der Pas de deux, dann das Ballet chinois pantomime, L'adoration de la pagode (Antoine Pitrot, Mimi Favier und Manon Rivière als Solisten, sowie 24 andere Tänzer als Figuranten) aufgeführt. Diese Ballette wurden während der folgenden Opernaufführungen wiederholt, lediglich die fünfte Vorstellung (7. November) wurde von anderen Titeln verziert. ${ }^{18}$ Choreograf all dieser Ballette war zweifellos der Hofballettmeister Antoine Pitrot, der maître des ballets, übrigens einer der ersten Vertreter der Gattung ballet d'action. Interessanterweise präsentierte Pitrot am 14. August 1755 im Pariser Théâtre Italien seine „chinesische“ Ballett-Pantomime „Les Tartares “, ${ }^{19}$ vielleicht von seiner Warschauer L'adoration de la pagode inspiriert oder eine Variante derselben. Der Komponist oder Arrangeur der Begleitmusik der Ballette in Warschau muss der erwähnte Johann Adam gewesen sein.

\section{Libretto}

Anlässlich der Premiere der L'eroe cinese in Warschau wurde hier das Opernlibretto herausgegeben und zwar in drei Sprachfassungen: der italienischen, der italienisch-französischen und der italienisch-deutschen ${ }^{20}$. Etwas später, nämlich 1755, erschien auch die polnische Übersetzung des Librettos von Józef Epifani Minasowicz, sie diente jedoch nur noch der moralisch belehrenden Lektüre. ${ }^{21}$

16 D-Dla, Nachlass Friedrich Christian, Nr. 2 c, Brief, in französischer Sprache, Augusts III. an Friedrich Christian, Warschau, 10. Oktober 1754.

17 D-Dla, Nachlass Maria Antonias, Nr. 10 a, Brief, in französischer Sprache, Heinrichs von Brühl an Maria Antonia, Warschau, 7. Oktober 1754.

18 D-Dla, OHMA G Nr. 61 b, fol. 79-105.

19 Les spectacles de Paris, ou Suite du Calendrier historique et chronologique des théatres. Cinquiéme partie pour l'année 1756, Paris; Duchesne, 1756, S. 155.

20 Alle drei in Drukarnia J.K.Mci i Rzeczypospolitej XX Scholarum Piarum, Warszawa 1754 gedruckt.

21 METASTASIO, Pietro. Bohatyr chiński, in: Zebranie rytmów, Bd. 4, Teil 1, Warszawa: Drukarnia J.K.Mci i Rzeczypospolitej XX Scholarum Piarum, 1755. 
Das chinesische Motiv des Librettos musste für die meisten Zuschauer der Warschauer Operalnia exotisch gewesen sein, obgleich Kontakte mit Ländern des Orients in der Adelsrepublik zur Staatraison gehörten und bspw. Besuche tatarischer oder türkischer Gesandter hier keine Seltenheit waren. Der chinesische Inhalt der Oper diente Metastasio lediglich zur Tarnung, den Absichten des immerhin ja kaiserlichen Dichters gemäß bezog er sich nämlich auf die Lage am Wiener Hof. Darüber hinaus wurde das weit entfernte China im aufgeklärten Europa zum Gegenstand nicht nur von Schilderungen von Reisenden und jesuitischen Missionaren sowie bedeutender Studien, sondern auch zu einem reizvollen und beliebten Thema verschiedener dramatischer Werke. Adrienne Ward erstellte eine reiche Liste europäischer dramatischer Werke mit chinesischer Thematik. ${ }^{22}$

In Metastasios Schaffen tauchte die chinesische Thematik schon 1735 in der einaktigen Festa teatrale Le cinesi auf. Die Musik dazu komponierte Antonio Caldara, der kaiserliche Vizekapellmeister. (Im Herbst 1754 kehrte dieses kleine Opus mit Textänderungen, die Metastasio vorgenommen hatte, und mit einer neuen Musik, die Christoph Willibald Gluck komponiert hatte, auf die Wiener Bühne zurück.)

Nach der chinesischen Thematik griff Metastasio auch 1752, als er das Dramma per musica L'eroe cinese schuf. In der chinesischen Entourage rechtfertigt der poeta cesareo wie gewöhnlich die hier auf dem Fundament der katholischen Religion basierende Macht der absoluten Monarchie. In der L'eroe cinese konzentriert er sich auf die extreme Loyalität des Untertanen gegenüber seinem Herrn und bedient sich der jesuitischen Glorifizierung des chinesischen Gehorsams, der als Ansatz der katholischen Fundamente begriffen wird. ${ }^{23}$ Der chinesische Titelheld ist Leango, ein hochrangiger Hofbeamter, der heroisch das Leben des eigenen Sohns opfert, um das Leben des Kaisersohns, des rechtmäßigen Erben des Reichs, zu retten. Und als das Volk Leango bittet, den Thron zu besteigen, stimmt er - als unterwürfiger Untergebener der Monarchie - nur zu, die Rolle des Regenten zu übernehmen.

Wie Metastasio in dem regelmäßig dem Druck eines Librettos vorausgehenden Argomento mitteilt, basiert der Inhalt der Oper auf der Geschichte Chao Kongs (in der Oper ist das Leango), die in den seinerzeit populären Fastes de la Monarchie chinoise des französischen Jesuiten Jean-Baptiste Du Halde erzählt wurde. ${ }^{24}$ Die von Du Halde geschilderte Episode beschreibt Ereignisse aus dem 9. Jahrhundert vor Christi Geburt.

Die Uraufführung der L'eroe cinese mit der Musik des kaiserlichen Komponisten Giuseppe Bonno fand am 13. Mai 1752 in Schönbrunn statt. Das diesem Spektakel gewidmete Libretto wurde nicht nur in Wien, sondern auch in politisch mit den Habsburgern verbundenen Städten - Neapel, Mailand, Palermo, Rom - veröffentlicht. Rasch wurde

22 WARD, Adrienne. Pagodas in Play: China on the Eighteenth-Century Italian Opera Stage. Lewisburg: Bucknell University Press, 2010, Appendix 1, S. 173-180.

23 W.o., S. 100.

24 DU HALDE, Jean-Baptiste. Description geographique, historique, chronologique, politique et physique de l'Empire de la Chine et de la Tartarie chinoise, Bd. 1-4, Paris: P. G. Lemercier, 1735. 
das Werk in ganz Europa berühmt. Mit der Musik verschiedener Komponisten wurde es in vielen Theatern aufgeführt. ${ }^{25}$

Bei Metastasio spielt die Handlung in Singana, der Hauptstadt der chinesischen Provinz Chensi. Das Bühnenbild umfasst hier standardgemäß wie in anderen damaligen Opere serie - und zwar ungeachtet des geografischen Orts der Handlung - das Interieur des Herrscherschlosses sowie des Hauptgotteshauses des Staates; einen abgelegenen und schattigen Platz im Schlosspark; die logge terrene des Schlosses, von denen aus in der Ferne eine Stadt, ein Fluss und Schiffe zu sehen sind. Dieses Mal sollte das alles jedoch in chinesischer Entourage gezeigt werden.

In der L'eroe cinese treten fünf Helden auf. Zwei davon haben ihre historischen Pendants: Leango, der Regent des chinesischen Kaiserreichs, ist Chao Kong, und Siveno, der vermeintliche Sohn Leangos und Geliebter Lisingas ist Suen Wang, der gerettete Sohn des Kaisers. Metastasio führte 3 weitere Personen ein: Lisinga, eine von den Chinesen gefangen gehaltene tatarische Prinzessin, die in Siveno verliebt ist, der ihre Liebe erwidert; Ulania, eine tatarische Prinzessin, Lisingas Schwester, die auch von den Chinesen gefangen gehalten wird, die Geliebte Minteos; Minteo, Mandarin des kaiserlichen Militärs, Ulanias Geliebter und Freund Sivenos, ein Findelkind, das Leango zusammen mit Siveno groß gezogen hat, in Wahrheit jedoch Leangos Sohn.

Worin besteht folglich der dramatische Konflikt? Leango muss die Untertanen davon überzeugen, dass die Herrschaft über das Kaiserreich nicht ihm zusteht, sondern dem rechtmäßigen Thronerben, mithin Siveno, der aber als tot gilt. Leango sucht Hilfe bei den Tataren, mit denen er gerade Frieden geschlossen hat. Zum Hauptmotiv des Dramas gehört auch die Situation der beiden tatarisch-chinesischen Liebespaare (Lisinga - Siveno, Ulania - Minteo), denen angesichts des Friedensschlusses die Trennung droht. Glücklich lösen sich jedoch alle Probleme, d.h. sowohl die staatlichen als auch die persönlichen, in dem für die Opera seria gängigen lieto fine in Wohlgefallen auf.

Das Warschauer Libretto ist im Prinzip eine getreue Nachbildung des Hubertusburger Librettos ${ }^{26}$, sogar die Textanordnung und die Seitenpaginierung wurden im Druck beibehalten. Dieselbe licenza, die August III. anlässlich seines Geburtstags glorifiziert und im Text die angeblichen Parallelen zwischen der l'eroe cinese und dem Jubilar hervorhebt, krönt das Werk in beiden Fällen. Es ist nicht bekannt, wer der Verfasser des Textes der licenza war, vielleicht Giovanni Ambroggio Migliavaccha, der Dichter des königlichkurfürstlichen Hofs. Die Musik der licenza stammte jedenfalls von Hasse, wovon ihre Anwesenheit in der Partitur des Werks zeugt.

Die einzige, dafür aber umso deutlichere Abweichung des Warschauer Librettos vom Hubertusburger bestand darin, dass die Arie der Lisinga „Se fra catene il core“ (II, 5) durch die Arie „Altro amor è il mio contenuto“ (Poet mir unbekannt) ersetzt wurde. Was war der Grund dafür? Schließlich interpretierte Albuzzi-Todeschini sowohl in Hubertusburg als auch in Warschau die Partie der Lisinga. Zweifellos wurde die Änderung jedoch auf ihren Wunsch hin vorgenommen. Es ist nicht ausgeschlossen, dass der Inhalt

25 Siehe SARTORI, Claudio. I libretti italiani a stampa dalle origini al 1800, Bd. 3. Cuneo: Bertola \& Locatelli Editori, 1991, S. 57-60.

26 METASTASIO, Pietro. L'eroe cinese. Dresda: Vedova Stössel, 1753. 
der neuen Arie eine dezente Anspielung auf die intime Verbindung der Künstlerin mit dem Minister von Brühl sein sollte. Jedenfalls hatten die Texte beider Arien eine andere Versstruktur, daher mussten auch ihre musikalischen Bearbeitungen (von Hasse?) verschieden sein.

\section{Musik}

Das Autograf der Partitur Hasses, die zweifellos der Uraufführung in Hubertusburg zugrunde lag, wird in der Biblioteca del Conservatorio di Musica in Mailand aufbewahrt. ${ }^{27}$ Die von einem Dresdner Kopisten ${ }^{28}$ angefertigte Hubertusburger Partitur hingegen und die dazu gehörigen Instrumentalstimmen sowie der Klavierauszug befinden sich in den Beständen der Sächsischen Landesbibliothek- Staats- und Universitätsbibliothek in Dresden. ${ }^{29}$ Eine einenartige Niederschrift des Werks in der Hubertusburger Fassung bildet eine Partitur, die keine Rezitative enthält und bisher in der Gegenstandsliteratur nicht erwähnt wurde. Sie wird in der Russischen Nationalbibliothek in St. Petersburg aufbewahrt $^{30}$ und befand sich seinerzeit höchstwahrscheinlich in Besitz der Schwester des künftigen Königs Stanislaus August Poniatowski Ludwika Zamoyska geb. Poniatowska.

Die der Oper vorausgehende Sinfonia besteht im Prinzip aus drei Teilen und ist also eine Art frühklassische Sinfonie. Dabei werden alle Instrumente, die an der Interpretation des Werks beteiligt sind, in Anwendung gebracht, also Flöten, Oboen, Hörner, Geigen, Bratschen und Instrumente, die zur Basslinie gehören - die in der Partitur in Worten vermerkten Violoncelli, Fagotte, das Kontrafagott (in einer der Arien als gran fagotto eingetragen), die Kontrabässe und zweifellos das Cembalo.

Leango ist in Hasses musikalischer Bearbeitung ein Tenor, die Partien der sonstigen Helden wurden im Sopranschlüssel, der auch die Alt-Tessitura beinhaltet, geschrieben. Auf die fünf Helden entfallen insgesamt 16 Arien - 6 im ersten und je 5 im zweiten und dritten Akt. Nur Lisinga, eindeutig die Primadonna, erhielt 4 Arien, darunter die den ersten Akt krönende herrliche aria di bravura. Die übrigen Helden singen je eine Arie in jedem der Akte. Die zusätzliche Arie in der licenza „In questo lieto giorno“ ist im Sopranschlüssel geschrieben, doch es ist nicht bekannt, wer von den Sängern sie ausgeführt hat. Den zweiten Akt schließt das Duett des Liebespaares Lisinga und Siveno „Perchè, se re tu sei“, das Finale der ganzen Oper hingegen ist selbstredend der coro dei solisti - „Sarà nota al mondo intero“, der zum Abschluss der Licenza wiederholt wird. In der ganzen Oper setzte Hasse nur 3 accompagnati ein und platzierte sie natürlich in Momenten besonderer dramaturgischer oder emotionaler Verstärkung (II, 6; III, 1; III, 7).

27 I-Mc, Part.Tr.Ms.160.

28 LANDMANN, Ortrun. Über das Musikerbe der Sächsischen Staatskapelle: Drei Studien zur Geschichte der Dresdner Hofkapelle und Hofoper anhand ihrer Quellenüberlieferung in der SLUB Dresden. Dresden, 2009, URL: http:/ / nbn:resolving.de/urn:nbn:de:bsz:14-qucosa-38515, S. 28, bezeichnet ihn als Kopisten x5.

29 D-Dl, Mus. 2477-F-73, Mus. 2477-F-74, Mus. 2477-F-75.

30 RUS-SPsc, F. 1021 Sobr. jed. muz. post. Op. 3, N. 22. 
L'eroe cinese ist ein typisches Werk für den Stil der Opera seria aus der Mitte des 18. Jahrhunderts. Dieser Stil wurde in hohem Maße eben von Metastasio und Hasse kreiert. Die Schematizität der musikalischen Konstruktion - das Wesen dieser Werke besteht schließlich nicht in der Originalität ihres Aufbaus, sondern in der Nuancen der Musikbearbeitung und in der Interpretationskunst der Sänger - führte dazu, dass alle Arien und das Duett den Aufbau einer fünfteiligen Arie da capo haben. Hasse hob sie jedoch mit musikalischen Mitteln fein voneinander ab. Er legte die Tempi der Arien - andantino, un poco lento, amoroso ma non troppo, lento, allegretto, allegro ma non troppo, allegro ma non presto, allegro assai, allegro, allegro di molto, vivace - genau fest. Die grundlegenden von ihm verwendeten Tonarten sind ausschließlich Dur-Tonarten (dreimal G-Dur, DDur, A-Dur und F-Dur, zweimal C-Dur, je einmal B-Dur und Es-Dur). Die Molltonarten - gleichnamige oder Paralleltonarten - werden mitunter in den mittleren Teilen, in denen auch Modulationen zu weiter entfernten Tonarten auftauchen, eingesetzt. Die Besetzung des die Arien begleitenden Orchesters, das selbstverständlich immer von der Gruppe der Instrumente, welche die Linie basso realisieren, unterstützt wird, ändert sich oft. Durch dieses Vorgehen wird die dramaturgische und emotionale Situation der Helden geschickt betont.

Die Oper verrät noch keine reformatorischen Merkmale, denen einige Jahre später sowohl Metastasio als auch Hasse unterliegen sollten. In der musikalischen Schicht passt sich das Werk dem Geschmack Augusts III. an. In den Arien glänzen die Sänger nicht nur durch Koloraturen, sondern auch durch ausgiebig verwendete Triller, Lombardund punktierte Rhythmen, große Intervallsprünge. Hasses Meisterschaft kommt nicht nur in der Fähigkeit zum Ausdruck, die Erwartungen des Mäzens zu erfüllen, sondern vor allem in der Schönheit der Musik, die präzis und subtil die dramatischen und emotionalen Wirrungen der Helden, die heute nicht immer bemerkt und gewürdigt werden, wiedergibt - übrigens nicht nur der Helden in den Hauptrollen, sondern auch in den Nebenrollen.

\section{Bibliography}

LANDMANN, Ortrun. Über das Musikerbe der Sächsischen Staatskapelle. Drei Studien zur Geschichte der Dresdner Hofkapelle und Hofoper anhand ihrer Quellenüberlieferung in der SLUB Dresden. Dresden, 2009, URL: http://nbn:resolving.de/urn:nbn:de:bsz:14-qucosa-38515, S. 28, bezeichnet ihn als Kopisten x5.

MELLACE, Raffaele. Johann Adolf Hasse. Palermo: L’Epos, 2004; idem. Johann Adolf Hasse. Neubearbeitete Ausgabe, Übersetzung Juliane Riepe. Beeskow: ortus musikverlag, 2016, S. 72.

WARD, Adrienne. Pagodas in Play: China on the Eighteenth-Century Italian Opera Stage, Lewisburg: Bucknell University Press, 2010.

ŻÓRAWSKA-WITKOWSKA, Alina. Johann Adolf Hassse und die Musik am polnischen Hof Augusts III. (1734-1763). In Hasse-Studien 7, Wolfgang Hochstein - Reinhard Wiesend (hrsg.). Stuttgart: Carus Verlag, 2012. 
ŻÓRAWSKA-WITKOWSKA, Alina. Muzyka na polskim dworze Augusta III [Musik am polnischen Hof Augusts III.], I. Teil. Lublin: Wydawnictwo Muzyczne Polihymnia, 2012.

ŻÓRAWSKA-WITKOWSKA, Alina. Between Dresden and Warsaw. The Travels of the Court of August III of Poland (Friedrich August II of Saxony). In Musicology Today: Polish Studies on Baroque Music, Szymon Paczkowski - Anna Ryszka-Komarnicka (eds.). Warsaw: Institut of Musicology University of Warsaw, 2009. 
